\title{
A Multiscale, Cell-Based Framework for Modeling Cancer Development
}

\author{
Yi Jiang \\ Theoretical Division, Los Alamos National Laboratory, Los Alamos, NM 87545, USA \\ jiang@lanl.gov
}

\begin{abstract}
We use a systems approach to develop a predictive model that medical researchers can use to study and treat cancerous tumors. Our multiscale, cell-based model includes intracellular regulations, cellular level dynamics and intercellular interactions, and extracellular chemical dynamics. The intracellular level protein regulations and signaling pathways are described by Boolean networks. The cellular level growth and division dynamics, cellular adhesion, and interaction with the extracellular matrix are described by a lattice Monte Carlo model. The extracellular dynamics of the chemicals follow a system of reactiondiffusion equations. All three levels of the model are integrated into a high-performance simulation tool. Our simulation results reproduce experimental data in both avascular tumors and tumor angiogenesis. This model will enable medical researchers to gain a deeper understanding of the cellular and molecular interactions associated with cancer progression and treatment.
\end{abstract}

\section{Introduction}

Since 2002, cancer has become the leading cause of death for Americans between the ages of 40 and 74 [1]. But the overall effectiveness of cancer therapeutic treatments is only $50 \%$. Understanding the tumor biology and developing a prognostic tool could therefore have immediate impact on the lives of millions of people diagnosed with cancer. There is growing recognition that achieving an integrative understanding of molecules, cells, tissues and organs is the next major frontier of biomedical science. Because of the inherent complexity of real biological systems, the development and analysis of computational models based directly on experimental data is necessary to achieve this understanding. Our model aims to capture knowledge through the explicit representation of dynamic biochemical and biophysical processes of tumor development in a multiscale framework.

Tumor development is very complex and dynamic. Primary malignant tumors arise from small nodes of cells that have lost, or ceased to respond to, normal growth regulatory mechanisms, through mutations and/or altered gene expression [2]. This genetic instability causes continued malignant alterations, resulting in a biologically complex tumor. However, all tumors start from a relatively simpler, avascular stage of growth, with nutrient supply by diffusion from the surrounding tissue. The restricted supply of critical nutrients, such as oxygen and glucose, results in marked gradients within the cell mass. The tumor 
cells respond both through induced alterations in physiology and metabolism, and through altered gene and protein expression [3], leading to the secretion of a wide variety of angiogenic factors.

Angiogenesis, formation of new blood vessels from existing blood vessels, is necessary for subsequent tumor expansion. Angiogenic growth factors generated by tumor cells diffuse into the nearby tissue and bind to specific receptors on the endothelial cells of nearby pre-existing blood vessels. The endothelial cells become activated; they proliferate and migrate towards the tumor, generating blood vessel tubes that connect to form blood vessel loops that can circulate blood. With the new supply system, the tumor will renew growth at a much faster rate. Cells can invade the surrounding tissue and use their new blood supply as highways to travel to other parts of the body. Members of the vascular endothelial growth factor (VEGF) family are known to have a predominant role in angiogenesis.

The desire to understand tumor biology has given rise to mathematical models to describe the tumor development. But no mathematical models of tumor growth yet can start from a single cell and undergo the whole process of tumor development. The state of the art in this effort employs hybrid approaches: Cristini et al. simulated the transition from avasular tumor growth to angiogenesis to vascular and invasive growth using an adaptive finite-element method coupled to a level-set method [4]; and Alarcon et al. used a hybrid of cellular automata and continuous equations for vascular tumor growth 56.

We have developed a multiscale, cell-based model of tumor growth and angiogenesis $[7 / 8$. This paper aims to review and promote this model framework. The Model section describes our model at three levels. The Parallelization section describes the hybrid scheme that makes the model a high-performance simulation tool. The Results section shows that the model reproduces quantitatively experimental measurements in tumor spheroids, and qualitatively experimental observations in tumor angiogenesis. We conclude by commenting on the broad applicability of this cell-based, multiscale modeling framework.

\section{$2 \quad$ Model}

Our model consists of three levels. At the intracellular level, a simple protein regulatory network controls cell cycle. At the cellular level, a Monte Carlo model describes cell growth, death, cell cycle arrest, and cell-cell adhesion. At the extracellular, a set of reaction-diffusion equations describes for chemical dynamics. The three levels are closely integrated. The details of the avascular tumor model has been described in 7 .

The passage of a cell through its cell cycle is controlled by a series of proteins. Since experiments suggest that more than $85 \%$ of the quiescent cells are arrested in the G1 phase [11, in our model, the cells in their G1 phase have the highest probability of becoming quiescent. We model this cell-cycle control through a simplified protein regulatory network [12, which controls the transition between G1 and S phases [7]. We model these proteins as on or off. By default this 
Boolean network allows the cell transition to $\mathrm{S}$ phase. However, concentrations of growth and inhibitory factors directly influence the protein expression. If the outcome of this Boolean regulatory network is zero, the cell undergoes cell-cycle arrest, or turns quiescent. When a cell turns quiescent, it reduces its metabolism and stops its growth.

The cellular model is based on the Cellular Potts Model (CPM) 910. The CPM adopts phenomenological simplification of cell representation and cell interactions. CPM cells are spatially extended with complex shapes and without internal structure, as domains on the lattice with specific cell ID numbers. Most cell behaviors and interactions are in terms of effective energies and elastic constraints. Cells evolve continuously to minimize the effective energy; the CPM employs a modified Metropolis Monte-Carlo algorithm, which chooses update sites randomly and accepts them with a Boltzmann probability. The total energy of the tumor cell system includes an interfacial energy between the cells that describes the cell-type dependent adhesion energy, a volume constraint that keeps the cell volume to a value that it "intends" to maintain; and an effective chemical energy that describes the cell's ability to migrate up or down the gradient of the chemical concentration. When the cell's clock reaches the cell cycle duration and the cell volume reaches the target volume, the cell will divide. The daughter cells inherit all properties of their parent with a probability for mutation.

Cells also interact with their environment, which is characterized by local concentrations of biochemicals. We consider two types of chemicals: the metabolites and the chemoattractants. The former includes nutrients (oxygen and glucose), metabolic waste (lactate), and growth factors and inhibitors that cells secret and uptake. The latter corresponds to the chemotactic signaling molecules. The chemicals follow the reaction-diffusion dynamics:

$$
\begin{aligned}
\frac{\partial M_{i}}{\partial t} & =d_{i} \nabla^{2} M_{i}-a_{i} . \\
\frac{\partial C_{i}}{\partial t} & =D_{i} \nabla^{2} C_{i}-\gamma C_{i}+B .
\end{aligned}
$$

Here the metabolite (concentration $M$ ) diffuses with the diffusion constant $d$ and is consumed (or produced) at a constant rate $a$. The chemoattractant, i.e. VEGF secreted by tumor cells to activate endothelial cells, diffuses with diffusion constant $D$ and decays at a rate $\gamma$. The local uptake function $B$ describes that an endothelial cell could bind as much as VEGF is available until its surface receptors saturate. Both the metabolic rates and the uptake function $B$ are functions of time and space. By assuming that (1) inside the tumor the diffusion coefficients are constant; and (2) each cell is chemically homogeneous, while different cells might have different chemical concentrations, we can solve the equations on a much coarser lattice than the lattice for CPM.

We use parameters derived from multicellular tumor spheroid experiments, the primary in vitro experimental model of avascular tumors 2]. Like tumors in vivo, a typical spheroid consists of a necrotic core surrounded by layers of quiescent and proliferating tumor cells, as shown in Fig. 2(c). It is critical to emphasize that 
this multicellular tumor spheroid experimental model recapitulates all major characteristics of the growth, composition, microenvironment, and therapeutic response of solid tumors in humans 2].

To model tumor angiogenesis, the simulation domain now corresponds to the stroma between the existing blood vessel and an avascular tumor. The tumor is a constant source for VEGF, whose dynamics follow Eqn. (3) and establish a concentration gradient across the stroma. Each endothelial cell becomes activated when the local VEGF concentration exceeds a threshold value. The activated vascular endothelial cells not only increase proliferation, decrease apoptosis rate, but also migrate towards the higher concentration of the VEGF signal.

The stroma consists of normal tissue cells and extracellular matrix (ECM). We model the ECM explicitly as a matrix of fibers. They are a special "cell" with a semi-rigid volume. The interstitial fluid that fills the space amongst the normal tissue cells and the fibers is more deformable than the fibers. When compressed by the growing endothelial sprout, the normal cells can undergo apoptosis and become part of the interstitial fluid. The endothelial cells can modify the local fibronectin concentration and re-organizing the fiber structure, as well as migrate on the matrix through haptotaxis, or follow the gradient of adhesion from the gradient of fibronectin density.

\section{Parallelization}

The underlying structure for the cell system is a $3 \mathrm{D}$ lattice. As all operations in the CPM are local, domain decomposition method is a natural scheme for parallelizing the CPM. We divide the physical domain into subdomains, one for each processor. Each processor then model the subsystem, storing only the data associated with the subdomain, and exchanging knowledge zone information with processors handling its neighboring subdomains. We adopt $1 \mathrm{D}$ domain decomposition based on two considerations. First, it will decrease the communication overhead when the knowledge zones are more continuous. Second, this simple decomposition allows us to store the cell information in two layers: the lattice layer and the cell information layer. As each cell occupies many lattice sites (over 100), this two-layer information structure far more efficient than storing all cell information on the lattice.

In the Monte Carlo update, to avoid expensive remote memory access, we use openMP, a parallel approach suited for shared memory processors. Before the Monte Carlo update of the lattice, the Master Node gathers subdomain lattice information and cell information from slave nodes. The Master Node performs Monte Carlo operations parallelly using openMP, and distributes subdomain data to corresponding slave nodes for next operation (Fig. 10 13.

We solve the reaction-diffusion equations that govern the chemical dynamics on a chemical lattice that is coarser than the cell lattice. This lattice is similarly decomposed and the equations solved in parallel within each subdomain. To accelerate long time simulations, we use implicit PDE sovling schemes based on BoxMG, an MPI-based multigrid PDE solver [14. 


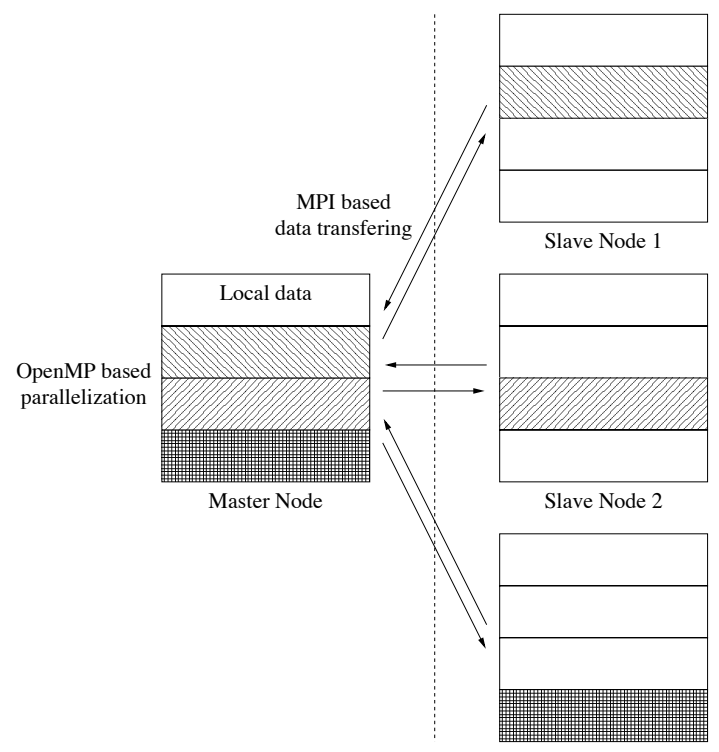

Slave Node 3

Fig. 1. OpenMP based parallelization in Monte Carlo operation

\section{Result}

In our simulations, a single tumor cell evolves into a layered structure consisting of concentric spheres of proliferating and quiescent cells at the surface and the intermediate layer respectively, and a necrotic core at the center of the spheroid, reproducing the experimental structure (Fig. 2).

Fig. 3 shows the comparison between the growth curves of a simulated solid tumor and two sets of spheroid experimental data. With $0.08 \mathrm{mM}$ oxygen and $5.5 \mathrm{mM}$ glucose kept constant in the medium, the number of cells (Fig. $3 \mathrm{a}$ ) and the tumor volume (Fig. 3 b) first grow exponentially in time for about 5-7 days.
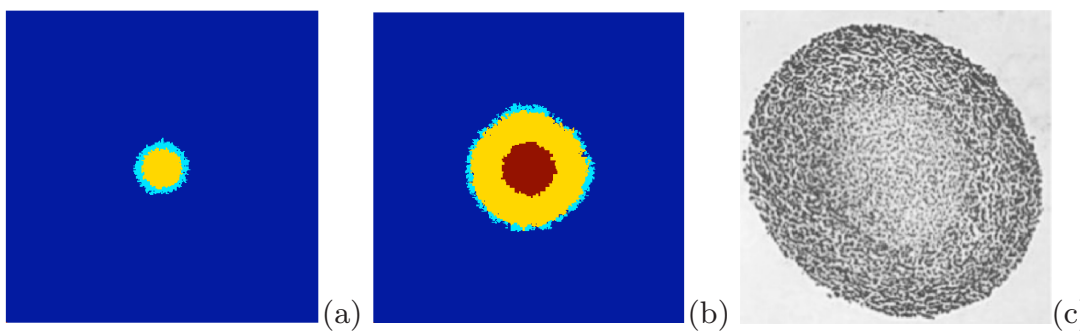

Fig. 2. Snapshots of a simulated solid tumor at 10 days (a), and 18 days (b) of growth from a single cell. Blue, cyan, yellow and crimson encode cell medium, proliferating, quiescent, and necrotic cells. (c) A histological crosssection of a spheroid of mouse mammary cell line, EMT6/R0. 
The growth then slows down, coinciding with the appearance of quiescent cells. In both the experiments [1516] and simulations, spheroid growth saturates after around 28-30 days. We fit both the experimental and the simulation data to a Gompertz function, in order to objectively estimate the initial doubling times and the spheroid saturation sizes [3. The doubling times for cell volume in experiments and simulations differ by a factor of two, over almost 5 orders of magnitude. The agreement was very good.
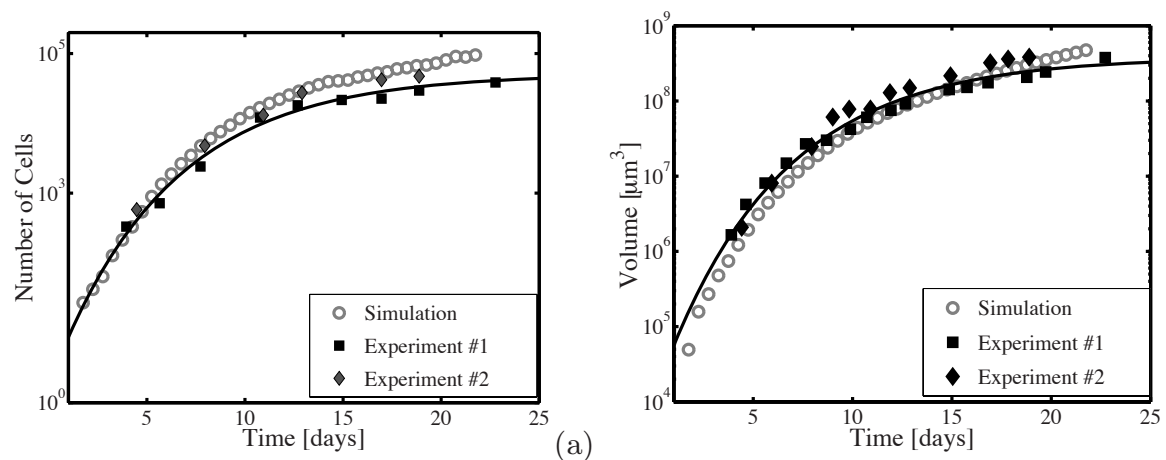

Fig. 3. The growth curves of a spheroid with $0.08 \mathrm{mM} \mathrm{O} 2$ and $5.5 \mathrm{mM}$ glucose in the medium: (a) the number of cells and (b) the volume of spheroid in time. The solid symbols are experimental data for EMT6/Ro 15 16]; the circles are simulation data. The solid lines are the best fit with a Gompertz function for experimental data.

In order to test the robustness of our model, we kept all the parameters in the model fixed at the values determined to produce the best fit to the growth of spheroids in $0.08 \mathrm{mM}$ oxygen and $5.5 \mathrm{mM}$ glucose. We then varied only the nutrient concentrations in the medium, as was done in spheroid experiments. Our simulations still showed good agreement between simulation and experimental growth curves when the external conditions were changed to $0.28 \mathrm{mM} \mathrm{O} 2$ and $16.5 \mathrm{mM}$ glucose in the medium [7.

In fitting our model to the experimental data, we predicted a set of conditions for the cell to undergo necrosis and the diffusion coefficients for the growth promoters and inhibitors to be in the order of $10^{-7}$ and $10^{-6} \mathrm{~cm}^{2} / \mathrm{hr}$, respectively. These predictions can be tested experimentally.

In tumor angiogenesis, Fig. 4 shows that our model is able to capture realistic cell dynamics and capillary morphologies, such as preferential sprout migration along matrix fibers and cell elongation, and more complex events, such as sprout branching and fusion, or anastomosis [8, that occur during angiogenesis. Our model constitutes the first cell-based model of tumor-induced angiogenesis with the realistic cell-cell and cell-matrix intereactions. This model can be employed as a simulation tool for investigating mechanisms, testing existing and formulating new research hypotheses. For example, we showed that freely diffusing VEGF would result in broad and swollen sprouts, while matrix bound VEGF typically generates thin sprouts [8, supporting the recent experimental interpretations. 


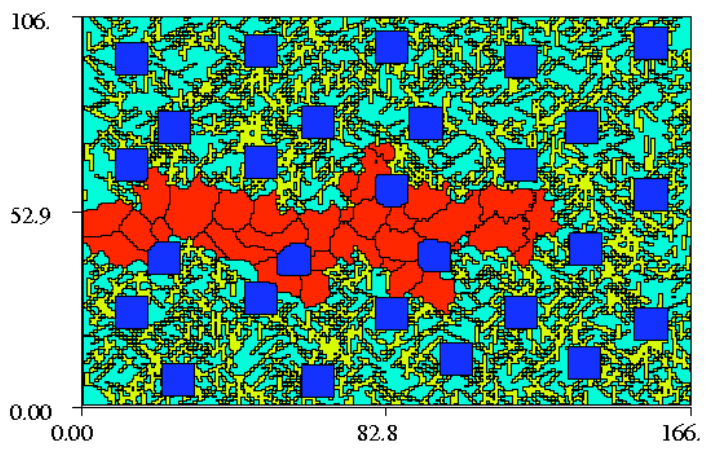

Distance toward Tumor - microns

Fig. 4. Tumor angiogenesis: a typical simulated vessel sprout. The left edge of the simulation domain is the blood vessel wall, while the right edge is the source of VEGF. Endothelial (red) cells grow and migrate in the stroma consists of normal cells (blue squares), matrix fibers (yellow) and interstitial fluids (green).

\section{Discussion and Outlook}

This multiscale approach treats cells as the fundamental unit of cancer development. We will further develop the model and investigate the growth of vessels into and inside tumor, as well as tumor growth and invasion. With this framework, we will model the development of cancer from beginning to full metastasis. We will also be able to test the effects of drugs and therapeutic strategies. Combined with the extant data (e.g. in vitro spheroid data and in vivo angiogenesis data), this type of model will help construct anatomically accurate models of a tumor and its vascular system. If successfully implemented, the model can guide experimental design and interpretation. Continuously revised by new information, the final model could potentially enable us to assess tumor susceptibility to multiple therapeutic interventions, improve understanding of tumor biology, better predict and prevent tumor metastasis, and ultimately increase patient survival. Furthermore, most biomedical problems involves systems level interactions. Genome, or molecular, or single cell studies cannot possibly provide systems level behaviors. This cell-based, multiscale modeling framework is applicable to a number of problems, e.g. biofilm formation, organogenesis, where cell-cell and cell-environment interactions dictate the collective behavior.

\section{Acknowledgments}

This work was supported by the National Nuclear Security Administration of the U.S. Department of Energy at Los Alamos National Laboratory under Contract No. DE-AC52-06NA25396. 


\section{References}

1. Jemal, A.:The Journal of the American Medical Association, 294 (2005) 12551259 .

2. Sutherland, R.M. Cell and environment interactions in tumor microregions: the multicell spheroid model. Science 240 (1988) 177-184.

3. Marusic, M., Bajzer Z, Freyer J.P., and Vuk-Pavlovic S: Analysis of growth of multicellular tumour spheroid by mathematical models. Cell Prolif. 27 (1994) 73.

4. Zheng, X., Wise S.M., and Cristini V.: Nonlinear simulation of tumor necrosis, neovascularization and tissue invasion via an adaptive finite-element/level-set method, Bull. Math. Biol. 67 (2005) 211-259

5. Alarcon, T., Byrne H.M., and Maini P.K.: A Multiple Scale Model for Tumor Growth, Multiscale Modeling and Simulation. 3 (2004) 440-475.

6. Alarcon, T., Byrne H.M., and Maini P.K.: Towards whole-organ modelling of tumour growth, Progress in Biophysics \& Molecular Biology. 85 (2004) 451 - 472.

7. Jiang, Y., Pjesivac J., Cantrell C, and Freyer J.P.: A multiscale model for avascular tumor growth, Biophys. J. 89 (2005) 3873-3883.

8. Bauer, A.L., Jackson T.L., and Jiang Y.: A cell-based model exhibiting branching and anastomosis during tumor-induced angiogenesis. Biophys. J. 92 (2007) in press.

9. Glazier, J.A. and Garner F: Simulation of the differential adhesion driven rearrangement of biological cells. Phys. Rev. E 47 (1993): 2128-2154.

10. Jiang, Y., Levine H. and Glazier J.A.: Differential adhesion and chemotaxis in mound formation of Dictyostelium, Biophys. J. 75(1998) 2615 -2625.

11. LaRue, K.E., Kahlil M., and Freyer J.P.: Microenvironmental regulation of proliferation in EMT6 multicellular spheroids is mediated through differential expression of cycline-dependent kinase inhibitors. Cancer Res. 64(2004) 1621-1631.

12. Data from the Kyoto Encyclopedia of Genes and Genomes (kegg.com).

13. He, K., Dong S., and Jiang Y.: Parallel Cellular Potts Model (2007) in preparation.

14. Austin T., Berndt M., et al. Parallel, Scalable, and Robust Multigrid on Structured Grids. Los Alamos Research Report (2003): LA-UR-03-9167.

15. Freyer, J.P. and Sutherland R.M.: Regulation of growth saturation and development of necrosis in EMT6/R0 multicellular spheroids by the glucose and oxygen supply. Cancer Res. 46(1986) 3504-3512.

16. Freyer, J.P. and R.M. Sutherland. Proliferative and clonogenic heterogeneity of cells from EMT6/Ro multicellular spheroids induced by the glucose and oxygen supply. Cancer Res. 46 (1986) 3513-3520. 